\title{
Chemosynthesis and photoelectric characterization of a novel solution-processable electrochromic copolymer based on alternating alkoxylating benzene and benzo[1,2-b:4,5-b']dithiophene derivatives
}

\author{
Zhen $\mathrm{Xu}^{1, \mathrm{a}}$, Jinsheng Zhao ${ }^{2, \mathrm{~b}}$ and Weiyu $\mathrm{Fan}^{1, \mathrm{c}}$ \\ ${ }^{1}$ State Key Laboratory of Heavy Oil Processing, China University of Petroleum (East China), \\ QingDao 266555, China \\ ${ }^{2}$ Shandong Key Laboratory of Chemical Energy Storage and Novel Cell Technology, Liaocheng \\ University, Liaocheng 252059, China \\ axz0215@126.com, bj.s.zhao@163.com, 'fanwyu@upc.edu.cn
}

\begin{abstract}
Keywords:Conjugated polymer, Chemical polymerization, Electrochromic, Spectroelectrochemistry, Benzo[1,2-b:4,5-b']dithiophene.
\end{abstract}

\begin{abstract}
The conjugated polymer poly (4 ,8 -bis ( (2-octyldodecyl) oxy) benzo [1,2-b:4,5-b'] dithiophene-co-p-didecyloxybenzene) (PBTB), was successfully chemically polymerized by reacting (4,8-bis((2-octyldodecyl)oxy)benzo[1,2-b:4,5-b']dithiophene-2,6-diyl)bis(trimethylstannane) with respective 2,5-didecyloxy-1,4-dibromobenzene in favorable yields via Stille cross-coupling reaction. Due to the incorporation of long alkoxy side chain, the resulting copolymer has high solubility in some common organic solvents. Besides, it was characterized by elemental analysis, gel permeation chromatography (GPC), cyclic voltammetry (CV) and UV-Vis-NIR spectroscopy. The electrochemical band-gap and optical band-gap was calculated to be $1.85 \mathrm{eV}$ and $2.03 \mathrm{eV}$ from $\mathrm{CV}$ curve and UV-Vis absorption spectrum, respectively. The PBTB film exhibited reversible electrochromic properties and rendered multi-colored display: orange-yellow reduced state $(0 \mathrm{~V})$, yellow-gray semi oxidized state $(1.15 \mathrm{~V})$ and gray fully oxidized state $(1.35 \mathrm{~V})$. In addition, kinetics study of the copolymer film revealed a maximum optical contrast $(\Delta \mathrm{T} \%)$ of $32 \%$, a fast response time of $0.4 \mathrm{~s}$ from the oxidized state to the neutral state and a high coloration efficiency of $267 \mathrm{~cm} 2 / \mathrm{C}$ at $\lambda \max$ of $510 \mathrm{~nm}$ in the visible region.
\end{abstract}

\section{Introduction}

The electrochromic phenomenon refers to a material or system which can reversibly change the optical properties (transmittance, reflectance, or absorbance) by altering its redox state at an applied external voltage [1]. In appearance, it showed reversible changes in color and transparency due to the formation of polaronic and bipolaronic bands causing a change in the spectral absorption [2]. Electrochromic material is considered to be one of the most promising smart materialssince they are potentially useful materials for a broad range of applications such as display devices, smart windows, glare-free mirror, military camouflage, etc.

Even though the inorganic materialsare the earlier studied active layers inelectrochromic devices and have been utilized over the past few decades, conjugated polymers has received much more attention because of their high optical contrasts, fast response times, easy processing, rich color change, easy molecular design and low cost. In these electrochromic conjugated polymers, polythiophenes aromatic heterocyclic, to be practical,has high degree of stability to oxygen and humidity, making its derivatives are more likely to be synthesized than other aromatic heterocyclic derivatives.At the same time, polythiophene and its derivatives have good redox reversibility and stability in the environmental [3]. Therefore, they displayed broad application prospects in the field of electrochromic.

Recently, a series ofthiophene-arylene-thiophene-like conjugated structures of polymers have been synthesized and usually present reasonable electrochromic properties [4,5]. However, their structural composition are usually simple, conjugated effects between the repeated aromatic heterocyclic are weak and because of their low solubility, the vast majority of them are obtained by 
the electrochemical polymerization method. Solvent-processable electrochromic polymer with excellent solubility, which canbe obtained by chemical polymerization in mass, thus reducing the cost and providing a strong competitive edge for the material commercialization, has become what researchers vigorously pursue.

To the best of our knowledge, long alkyl and alkoxy side chains connected to thiophene and benzene rings conjugated structure, stagger arrangement at the periphery of the main chain, thereby significantly increasing the solubility of the plane $\pi$-skeleton.According to above considerations, in this work, soluble poly ( 4,8 -bis ( (2- octyldodecyl) oxy ) benzo [ 1,2 - b : 4,5 -b' ] dithiophene-co-p-didecyloxybenzene) (PBTB) issynthesized by coupling reaction according to scheme 1.The spectroelectrochemical and electrochromic properties of the PBTB film are also investigated in details. The synthesized copolymer shows multi-colors at different applied voltages with fast response time, good optical contrast and high coloration efficiency.
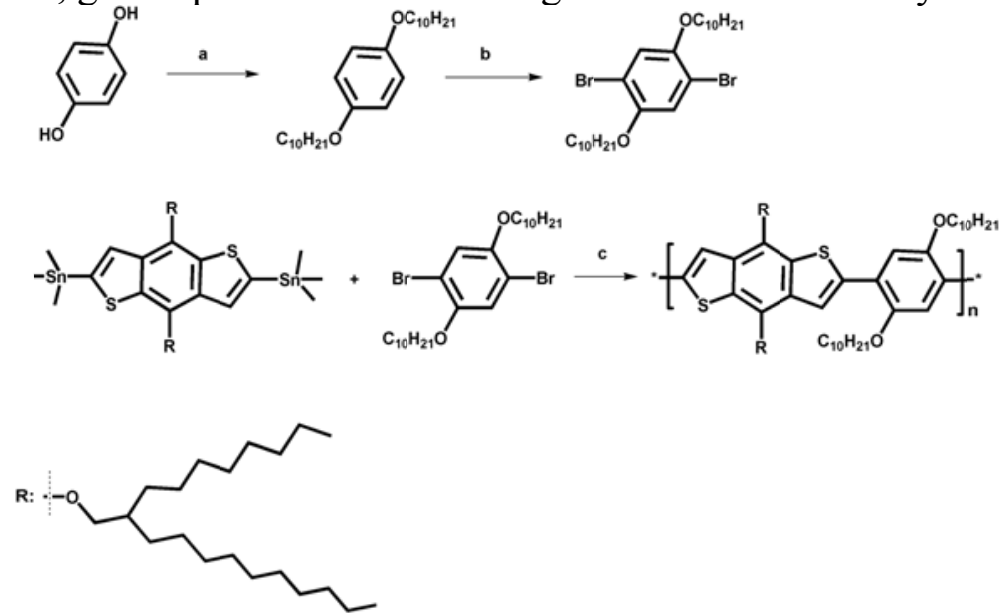

Scheme 1.Synthetic route of the polymer. (a) DMF, K2CO3, 1-bromine decane, TBAB, $120^{\circ} \mathrm{C}, 90$ min. (b) $\mathrm{HBr}, \mathrm{Br} 2,135^{\circ} \mathrm{C}, 5 \mathrm{~h}$. (c) toluene, $\mathrm{Pd}(\mathrm{PPh} 3) 2 \mathrm{Cl} 2$, reflux, $48 \mathrm{~h}$.

\section{Experimental section}

\subsection{Materials and Instrumentation.}

All the chemicals and reagents are purchased from Sigma-Aldrich chemical company except that(4,8-bis((2-octyldodecyl)oxy)benzo[1,2-b:4,5-b']dithiophene-2,6-diyl)bis(trimethylstannane) is from Derthon Optoelectronic Materials Science Technology Co LTD. And the reagents adopted in the experiment are all chemical grade and have been directly used without further purification. 1,4didecyloxybenzene[6] and 2,5-didecyloxy-1,4-dibromobenzene[7] were prepared according to the previously reported literature methods.

Elemental analyses are carried out on a Thermo Finnigan Flash EA 1112, CHNS-O elemental analysis instrument. Molecular weight of the polymer are determined by Waters 1515-2414 gel permeation chromatography (GPC). Electrochemical cyclic voltammetry performance test is performed on a CHI $760 \mathrm{C}$ workstation with ITO/glass as transparent working electrode (WE), platinum wire as the counter electrode, and $\mathrm{Ag}$ wire (-0.03 V vs. SCE.) as the pseudo-reference electrode immersed in $0.1 \mathrm{M} \mathrm{TBAHF}_{6} / \mathrm{ACN}$ as the supporting electrolyte. Spectroelectrochemical and kinetic measurements are recorded on a Varian Cary $5000 \mathrm{UV}-$ Vis-NIR spectrophotometer connected with the above electrochemical workstation. Photos of the polymer film are taken with a Canon Power Shot A3000 IS digital camera.

\subsection{Synthesis of $\pi$-conjugated polymer PBTB.}

In turn, (4,8 -bis ( ( 2-octyldodecyl) oxy)benzo [1,2-b:4,5-b'] dithiophene-2,6-diyl) bis (trimethylstannane) (0.407 g), 2,5-didecyloxy-1,4-dibromobenzene (0.201 g), $\mathrm{Pd}\left(\mathrm{PPh}_{3}\right)_{2} \mathrm{Cl}_{2}(0.015 \mathrm{~g})$ and $100 \mathrm{~mL}$ toluene were added into $250 \mathrm{~mL}$ single-necked flask. The mixture was refluxed under argon atmosphere for $48 \mathrm{~h}$. Excess solvent was evaporated, and then a large amount of methanol solvent was added to give the suspension. The polymer obtained by filtration, which was further purified by Soxhlet extraction with $\mathrm{MeOH}$ and acetone to remove the catalyst and oligomer, and 


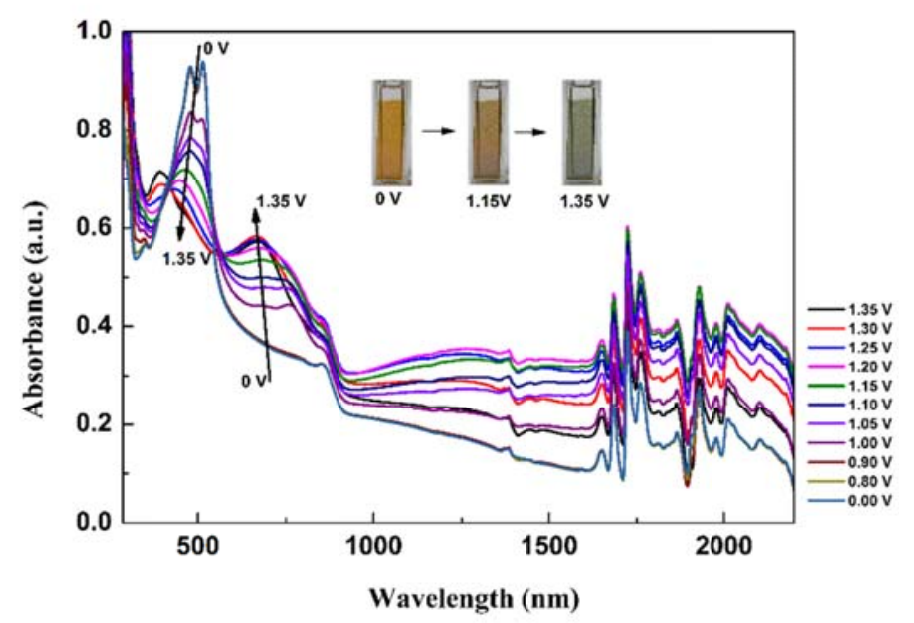

Figure 2. Electronic absorption spectra and the color changes of PBTB film at different potentials 3.3 Electrochromic switching studies.

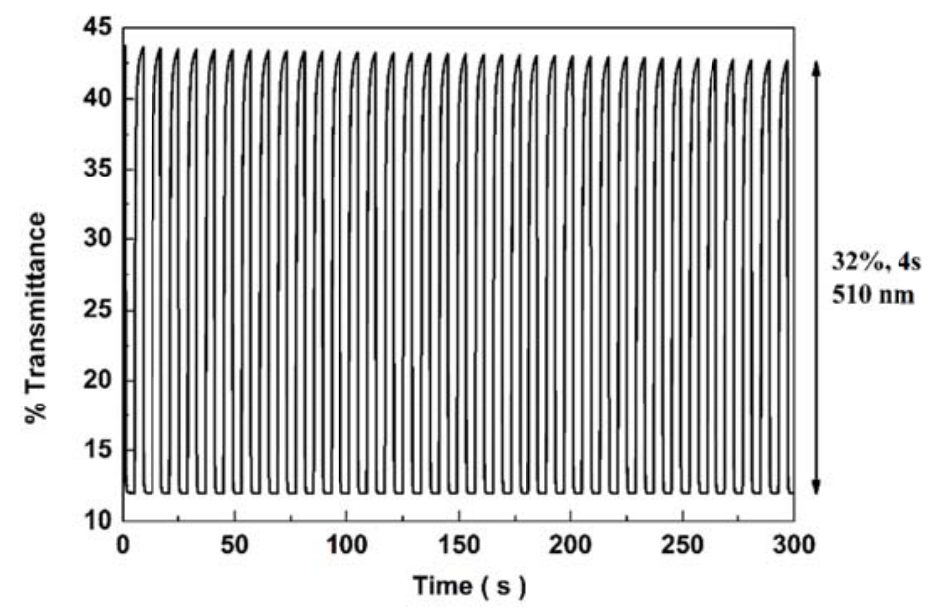

Figure 3.Electrochromic switching, percent transmittance change monitored at $510 \mathrm{~nm}$ for PBTB between 0 and $1.35 \mathrm{~V}$

Switching properties of PBTB film were also measured at the maximum absorption in visible regions. As shown in figure 3, it revealed optical contrast value of $32 \%$ at $510 \mathrm{~nm}$ with a response time of $0.4 \mathrm{~s}$. The coloration efficiency (CE) of PBTB film was calculated to be $267 \mathrm{~cm}^{2} / \mathrm{C}$.

\section{Summary}

In summary, a novel $\pi$-conjugated polymers poly $(4,8$-bis((2-octyldodecyl)oxy) benzo [1,2-b:4,5-b'] dithiophene-co-p-didecyloxybenzene) (PBTB) was synthesized by chemical polymerization. The PBTB film showed three colors (orange-yellow, yellow-gray and gray) at different applied voltages. In addition, the high optical contrast (32\%), fast switching time (0.4 s) and satisfactory coloration efficiency $\left(267 \mathrm{~cm}^{2} / \mathrm{C}\right)$ make the polymer good candidate for electrochromic display application.

\section{Acknowledgements}

The work was financially supported by the Postgraduate Innovation Project of China University of Petroleum (East China) (YCX2015022) and the Fundamental Research Funds for the Central Universities (15CX06049A). 


\section{References}

[1] B. C. Thompson, P. Schottland, J. R. Reynolds, et al. In Situ Colorimetric Analysis of Electrochromic Polymers and Devices. Chemistry of Materials. 12 (2000) 6, 1563-1571.

[2] J. L. Bredas, R. R. Chance, R. Silbey. Comparative theoretical study of the doping of conjugated polymers: Polarons in polyacetylene and polyparaphenylene. Physical Review B. 26(1982)10, 5843-5854.

[3] E. Sahin, P. Camurlu, L. Toppare, et al. Synthesis and characterization of thiophene functionalized polystyrene copolymers and their electrochemical properties. Polymer International. 54 (2005)12, 1599-1605.

[4] B. Wang, J. S. Zhao, C. S. Cui, et al. Electrochromic properties of a novel low band gap conjugated copolymer based on 1,4-bis(2-thienyl)-naphthalene and 3,4-ethylenedioxythiophene. Electrochimica Acta. 56 (2011)13, 4819-4827.

[5] O. Atwani, C. Baristiran, A. Erden, et al. A stable, low band gap electroactive polymer: Poly(4,7-dithien-2-yl-2,1,3-benzothiadiazole). Synthetic Metals. 158 (2008)3, 83-89.

[6] B. Mohr, V. Enkelmann, G. Wegner. Synthesis of alkyl- and alkoxy-substituted benzils and oxidative coupling to tetraalkoxyphenanthrene-9,10-diones. The Journal of Organic Chemistry. 59 (1994)3, 635-638.

[7] B.-L. Lee, T. Yamamoto. Syntheses of New Alternating CT-Type Copolymers of Thiophene and Pyrido[3,4-b]pyrazine Units: Their Optical and Electrochemical Properties in Comparison with Similar CT Copolymers of Thiophene with Pyridine and Quinoxaline. Macromolecules. 32(1999)5, 1375-1382. 